DOI: https://doi.org/10.46296/yc.v4i7.0031

\title{
LAS MICROFINANZAS Y SU APORTE AL DESARROLLO PRODUCTIVO LOCAL. UN ENFOQUE DE GÉNERO
}

\section{MICROFINANCE AND ITS CONTRIBUTION TO LOCAL PRODUCTIVE DEVELOPMENT. A GENDER APPROACH}

\author{
Flores-Anchundia, Nancy Paola1*; Valdés-Pérez, Dayana ${ }^{2}$ \\ ${ }^{1}$ Maestrante en Administración de Empresas con mención en Calidad y Productividad de la \\ Pontificia Universidad Católica del Ecuador Sede Manabí, PUCESM. Portoviejo, Ecuador. \\ Orcid: https://orcid.org/0000-0001-6078-9064 \\ ${ }^{2}$ Coordinadora de la Maestría en Administración de Empresas con mención en Calidad y \\ Productividad de la Pontificia Universidad Católica del Ecuador Sede Manabí, PUCESM. \\ Portoviejo, Ecuador. Orcid: https://orcid.org/0000-0002-5063
}

*Correo: nflores1812@pucesm.edu.ec

\begin{abstract}
Resumen
Las microfinanzas son estrategias que permiten responder y actuar frente a la pobreza y generar desarrollo productivo en los territorios locales, puesto que abarcan la intermediación financiera y la intermediación social, lo que implica que además de entregar servicios bancarios, permiten generar un aporte al crecimiento sostenible de los grupos de interés social. En la presente investigación se analizó la incidencia de las microfinanzas sobre el desarrollo productivo desde un enfoque de género; así mismo se evaluó si los microfinanciamientos han contribuido al desarrollo del sector productivo del país, disminuyendo los obstáculos causados por el género, debido a que no se considera la equidad de género, ni entre sus objetivos ni entre las condiciones para su desempeño. El estudio se delimitó en la ciudad de Portoviejo y los sectores rurales del mismo, involucrando a las mujeres microempresarias del sector. Por lo tanto, se aplicaron encuestas a 328 mujeres que han recibido apoyo o financiamiento de entidades bancarias, de manera que se logró inferir acerca del impacto del emprendimiento sobre el empoderamiento femenino. Se determinó que las microfinanzas tienen una importancia relevante en el empoderamiento femenino, puesto que si bien han sido concebidas para generar desarrollo productivo; los grupos de mayor vulnerabilidad, en algunos casos, no podían acceder a estas fuentes de financiamiento. Esto se debe a que las mujeres señalan en su gran mayoría que debido al emprendimiento que han liderado, se genera una satisfacción personal con una significancia altamente relacionada con el empoderamiento personal y colectivo.
\end{abstract}

Palabras clave: mujeres, microfinanzas, créditos bancarios, desarrollo productivo, emprendimiento.

\begin{abstract}
Microfinance is a strategy that allows responding and acting against poverty and generating productive development in local territories, since it encompasses financial intermediation and social intermediation, which implies that in addition to providing banking services, they allow generating a contribution to sustainable growth of social interest groups. The present investigation analyzed the incidence of microfinance on productive development from a gender perspective; Likewise, it was evaluated whether microfinance has contributed to the development of the country's productive sector, reducing the obstacles caused by gender, since gender equity is not considered, neither among its objectives nor among the conditions for its performance. The study was delimited in the city of Portoviejo and its rural sectors, involving women microentrepreneurs in the sector. Therefore, surveys were applied to 328 women who have received support or financing from banking entities, so that it was possible to infer about the impact of entrepreneurship on female empowerment. It was determined that microfinance has a relevant importance in female empowerment, since although they have been conceived to generate productive development; the most vulnerable groups, in some cases, could not access these sources of financing. This is because the majority of women point out that due to the entrepreneurship they have led, personal satisfaction is generated with a significance highly related to personal and collective empowerment.
\end{abstract}

Keywords: women, microfinance, bank loans, productive development, entrepreneurship.

Información del manuscrito:

Fecha de recepción: 02 de junio de 2020

Fecha de aceptación: 08 de julio de 2020

Fecha de publicación: 10 de julio de 2020 


\section{Introducción}

La pobreza es un fenómeno, problema o condición social de naturaleza multidimensional, cuyos factores, consecuencias y variables asociadas son tan diversos como los lugares o regiones del mundo que la padecen (Colmenares, 2017). En 2017, el número de personas viviendo en la pobreza llegó a 184 millones $(30,2 \%$ de la población mundial), de los cuales 62 millones se encontraban en la extrema pobreza $(10,2 \%$ de la población, el porcentaje más alto desde 2008), de acuerdo con el informe Panorama Social de América Latina 2018, expuesto por la Secretaría Ejecutiva del organismo regional de las Naciones Unidas, en Chile.

Según el Instituto Nacional de Estadísticas y Censos (INEC, 2018) para junio del año 2018, el Ecuador tuvo una línea de pobreza en $\$ 84,72$ mensuales por persona y de extrema pobreza en $\$ 47,74$ mensuales por persona. Bajo este umbral, la incidencia de pobreza en el Ecuador es del $24,5 \%$, mientras que la extrema pobreza es del $9 \%$. Para diciembre del 2019 la pobreza se ubicó en $25 \%$ y la pobreza extrema en 8,9 ; es decir no se han presentado cambios estadísticamente significativos (INEC, 2019). Se evidencia que, si bien la pobreza y extrema pobreza se mantiene constante en el período reportado, la pobreza alcanza niveles altos en nuestro país.

El desarrollo productivo debe ser entendido en función de sus múltiples dimensiones: social, económica, política, institucional, ambiental y cultural. En efecto, la pobreza, no debe ser asociada tan solo a la existencia de necesidades básicas insatisfechas o escasos ingresos económicos; también debe relacionarse con la falta de acceso equitativo a los activos $y$ a las oportunidades que oferta la sociedad bajo diferentes mecanismos de cooperación económica (Mballa, 2017). El desarrollo del sector productivo es un elemento fundamental para mejorar $y$ optimizar la competitividad de una nación, generando un aceleramiento en el incremento económico sostenido en el largo plazo y en la mejora continua de las condiciones sociales a través de la generación de empleo y valor agregado en la 
economía (Ministerio de Economía y Finanzas de Perú, 2018). La creación de empleo y la inclusión económica y social son factores de suma importancia en el crecimiento o desarrollo productivo de una región, país o localidad. No obstante, el desarrollo local se fundamenta en la identificación y aprovechamiento de las fortalezas y potencialidades endógenas, como estrategia de atención a las necesidades sociales más apremiantes. Históricamente, estas necesidades han sido evaluadas en función de las capacidades humanas, accesibilidad a la infraestructura, condición de vulnerabilidad, ingresos y exclusión (García-Lobo, 2004).

Las microfinanzas son estrategias que permiten responder y actuar frente a la pobreza y generar desarrollo productivo en los territorios locales, puesto que los financiamientos pequeños abarcan la intermediación financiera y la intermediación social, lo que implica que además de entregar servicios de crédito, ahorro y seguro, también aportan con el crecimiento sostenible y la formación de grupos de interés social (Banco Mundial, 2012). Por tanto, el microfinanciamiento debe ser integral, y generar un aporte significativo en las necesidades financieras de la comunidad (Iglesias, 2008). Específicamente, el microcrédito es un mecanismo concreto de implementar las microfinanzas en un determinado sector, que por sus condiciones sociales requiere del apoyo financiero para iniciar o continuar con sus actividades económicas.

La FAO (2002) menciona que las microfinanzas pueden generar efectos adversos en la población, cuando no se consideran los límites y desafíos de esta actividad, por consiguiente, dicho proceso debe encontrar un equilibrio entre el acceso al servicio financiero y la estabilidad, protección e integridad de la persona o colectivo que recibe el financiamiento. Las microfinanzas deben orientarse hacia la atención de las necesidades, en las diferentes dimensiones que involucran estos requerimientos. Además, una racionalización de pequeñas finanzas genera nuevas fórmulas de economía social distantes del tradicional endeudamiento y empobrecimiento generalizado por el sistema financiero. Se busca desarrollar estrategias productivas 
con sistemas de administración avanzados, según los lineamientos de cooperativas y sociedades productivas. Esto implica articular los mecanismos que doten a la ciudadanía en desventaja de una financiación adecuada para su actividad emprendedora (Mballa, 2017).

Por su parte, la participación de las mujeres en el mercado laboral ha crecido sostenidamente durante las últimas décadas, su aporte económico ha cobrado cada vez más importancia fuera del ámbito de la reproducción social. Además de incorporarse como empleadas $u$ ocupadas por cuenta propia, muchas mujeres han optado por el autoempleo y los micro y pequeños emprendimientos productivos y comerciales. Sin embargo, las diferencias de género han subsistido, y las mujeres siguen siendo negativamente afectadas en términos de inserción laboral, permanencia y calidad del empleo, y por lo tanto, en sus posibilidades de acceso a la independencia económica y la autonomía (CEPAL, 2005).

El propósito de la presente investigación es analizar la incidencia de las microfinanzas y como estos financiamientos pequeños han contribuido al desarrollo del sector productivo del país, disminuyendo los obstáculos causados por el género, debido a que no se considera la equidad de género, ni entre sus objetivos ni entre las condiciones para su desempeño. Además, las unidades empresariales orientadas hacia las mujeres se han promovido sobre todo en el marco de los programas de generación de ingresos a través de la creación de microempresas, puesto que con frecuencia no logran la sustentabilidad $\sin$ los apoyos provenientes de dichos programas. El desarrollo productivo ha facilitado la integración de las mujeres y la creación de mayores oportunidades para acceder a sus beneficios.

\section{Metodología}

\subsection{Tipo de Investigación}

El presente trabajo de investigación dado su naturaleza documental cualitativa, se realizó bajo una investigación histórica-analítica que busca obtener información de los aspectos centrales, con el objeto de conocer los procesos de inclusión financiera de la mujer ecuatoriana, los límites que se presentan cuando 
desean acceder al crédito y el comportamiento de las entidades financieras ante el desafío de la inclusión de la mujer en el sector productivo. Además de un enfoque cuantitativo por medio de la aplicación de encuestas, para ello se determinó una muestra representativa de personas que deben ser encuestadas partiendo de la población total 0 universo considerado en esta investigación.

El estudio se delimitó en la ciudad de Portoviejo y los sectores rurales del mismo, involucrando a las mujeres microempresarias del sector. Es necesario aplicar técnicas $\mathrm{e}$ instrumentos que permitan establecer un contacto con el entorno en el que se está investigando.

Adicionalmente, se debe mencionar que se realizó una investigación bibliográfica-documental, dado que para lograr los objetivos planteados se utilizó información científica propuesta por organismos $\mathrm{e}$ instituciones relacionadas con la administración de bancos privados; para establecer si un microcrédito permite a una mujer lograr el empoderamiento 0 conocer las diferencias del estado actual con respecto al problema de estudio.

\subsection{Diseño y Muestra}

El diseño de la investigación que se implementó es de tipo descriptivo, puesto que permitió considerar el fenómeno de estudio y sus componentes, también la asociación de variables que nos permitan cuantificar los datos, y conocer la relación existente entre el acceso a microcréditos y el empoderamiento en mujeres emprendedoras. Además, permitió explicar las características del empoderamiento de la mujer, dado que describe tendencias de un grupo o población.

La muestra fue tomada en la ciudad de Portoviejo y determinada mediante la unidad de análisis como son las mujeres microempresarias que han recibido financiamiento del Banco Guayaquil y con los datos presentados por la Superintendencia de Bancos, mediante muestras probabilísticas de forma aleatoria para que todas las microempresarias puedan tener la oportunidad de ser elegidas.

El cálculo del tamaño de la muestra es uno de los aspectos a concretar 
en las fases previas de la investigación comercial y determina el grado de credibilidad que se concede a los resultados obtenidos.

La fórmula empleada para calcular el tamaño de muestra fue obtenida de Aguilar-Barojas (2005).

$$
\boldsymbol{n}=\frac{N * z^{2} * p * q}{e^{2}(N-1)+z^{2} * p * q}
$$

Donde:

N: 3000. Corresponde al tamaño de la población o universo.

z: 1,96. Es una constante que depende del nivel de confianza asignado. El nivel de confianza indica la probabilidad de que los resultados de la investigación tengan certeza.

e: 0,05: Es el error muestral deseado. El error muestral es la diferencia existente entre el resultado obtenido mediante la aplicación en la muestra y el que se obtendría si se considerara el total de la población.

p: 0,4. Es la proporción de individuos que poseen en la población la característica de estudio.

q: 0,6. Es la proporción de individuos que no poseen esa característica, es decir, es 1-p. n: es el tamaño de la muestra.

$$
n=\frac{1.96^{2} \times 0.4 \times 0.6 \times 3000}{\left(0.05^{2} \times(3000-1)\right)+1.96^{2} \times 0.4 \times 0.6}
$$

Ecuación 1. Fórmula para determinar tamaño de la muestra

El propósito de la presente fórmula es obtener una muestra representativa de la población, para disminuir los recursos financieros empleados en el desarrollo de la investigación, dado que el universo de la investigación es muy amplio.

Para recopilar información se realizaron encuestas a las mujeres microempresarias del cantón Portoviejo, Manabí, Ecuador, de manera que se pueda inferir acerca de si una mujer luego de tomar un crédito de inversión aumenta su empoderamiento.

La información obtenida fue analizada a través de tablas y gráficos que facilitaron la interpretación de los resultados.

\section{Resultados y discusión}

\subsection{Percepción de las emprendedoras}


La figura 1 refleja la percepción de las participantes en la investigación respecto al estatus económico en el que se encuentran, evidenciando que el $48,9 \%$ de la muestra considera que se encuentra en clase media, el 34,6\% en clase media baja, mientras que el $11 \%$ en clase baja. Se demuestra que aproximadamente la mitad de la población se encuentra en clase media; por otra parte, en la Encuesta de Estratificación del Nivel Socioeconómico (NSE) del Instituto Nacional de Estadísticas y Censos (INEC, 2011) se determinó que el $22,8 \%$ de la población ecuatoriana se encuentra en la estratificación $\mathrm{C}+$, que en una escala de 5 valores (A, $B$, C+, C-, D), representa la media de la clasificación, por lo tanto se asocia con la clasificación de clase media, propuesta en la presente investigación. Se debe considerar que según el INEC el piso de las viviendas de las personas que se encuentran en este estrato económico es de cerámica, baldosa, vinil o marmetón; además, el $83 \%$ de los hogares dispone de servicio de teléfono convencional y el $96 \%$ de los hogares cuenta con una refrigeradora. En lo que respecta a la educación, en promedio el jefe del hogar tiene un nivel de instrucción secundaria completa, asimismo, estos jefes de hogar se desempeñan como trabajadores de servicios, comerciantes y operadores de instalación de máquinas y montadores. El $77 \%$ de los hogares está afiliado o cubierto por el Seguro del IESS (seguro general, seguro voluntario o campesino) y/o seguro del ISSFA o ISSPOL.

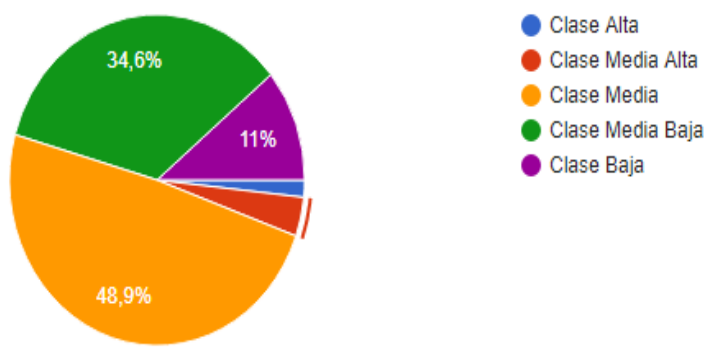

Figura 1. Percepción del estatus económico

En lo que respecta al emprendimiento y a la capacidad de generar negocios propios, se determinó que el $89 \%$ de las participantes lo han realizado (figura 2). Camino \& Aguilar (2017) mencionan que en el "análisis a nivel regional se puede describir al emprendimiento como una buena opción de carrera en la región Latinoamericana y el Caribe, puesto que tiene apenas un $17 \%$ de aceptación entre los adultos en edad de trabajar; en Europa el $79 \%$ de los 
países bajos lo consideran relevante; mientras que en Asia menos del $40 \%$ lo determinan como buena opción de carrera; los contrastes señalados dan un reflejo de la realidad económica de las regiones en función a la percepción del emprendimiento y su mantenimiento en el tiempo, los mismos que se ven reflejados en función a sus economías delimitando tres segmentos marcados: por la explotación de materias primas, la eficiencia en la productividad y el desarrollo tecnológico e innovación" (pág. 77).

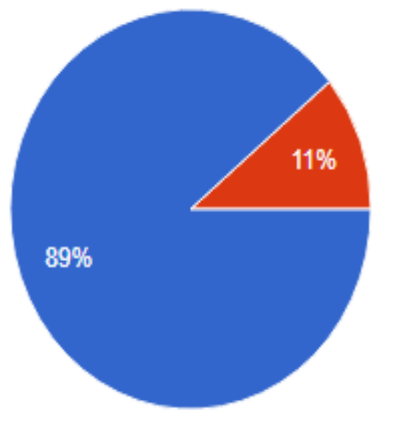

Figura 2. Negocios propios

Asimismo, la figura 3 evidencia que los emprendimientos han impactado positivamente las condiciones de vida, de modo que el $91,6 \%$ de las mujeres está de acuerdo con esta aseveración. De acuerdo con el Global Entrepreneurship Monitor (GEM, 2015), en el año 2015
Ecuador aumentó su Tasa de Actividad Emprendedora (TEA) en un punto respecto al año anterior, señalando que en el $68,8 \%$ de los casos los emprendedores deciden optar este camino debido a que no tienen una mejor opción de trabajo.

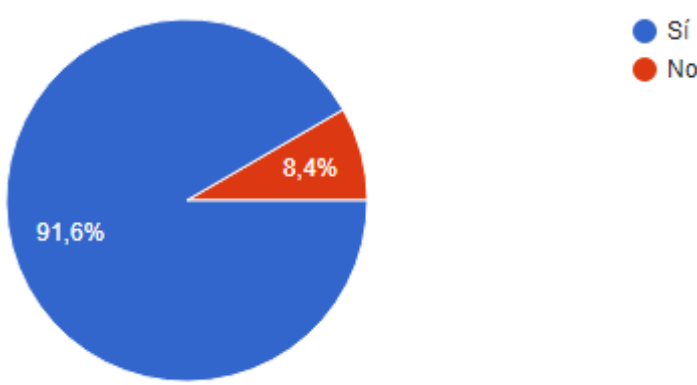

Figura 3. Efectos del emprendimiento sobre su condición de vida

No obstante del crecimiento y las altas tasas de emprendimiento a temprana edad que se registran en Ecuador, superando los promedios para la región latinoamericana, en concordancia con las estadísticas expuestas por el GEM (2017) es evidente que aproximadamente la mitad de los emprendimientos tienen menos de 5 años, por lo tanto, aún están pasando por momentos de incertidumbre que los pueden llevar a cerrar sus negocios (figura 4). Adicionalmente, la presente investigación comprueba que prácticamente todos los emprendimientos presentan 
dificultades para sostenerse en el mercado actual, debido principalmente a la falta de financiamiento $(54,2 \%)$ falta de clientes $(25,2 \%)$, y en menor medida por la falta de apoyo familiar y falta de apoyo de la pareja, tal como lo muestra la figura 5. La pandemia del COVID-19 es un factor que combinado con los antes mencionados, configura un escenario complicado de sobrellevar para todos los emprendimientos que se han generado antes y durante la pandemia.
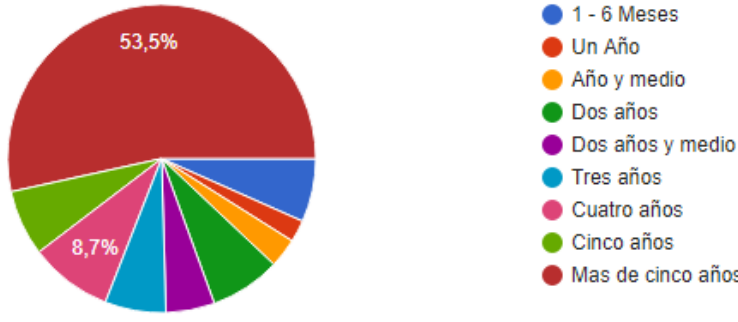

Figura 4. Tiempo de antigüedad del negocio
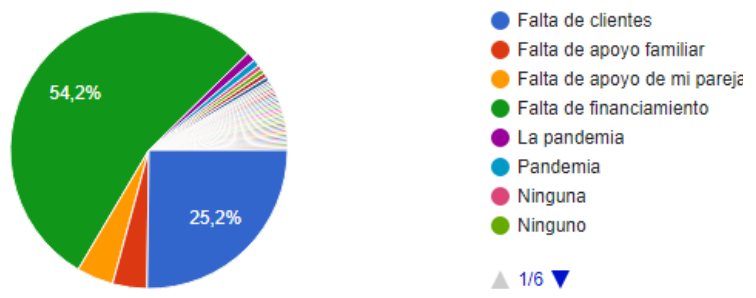

Figura 5. Dificultades para mantener el negocio

La figura 6 muestra las fuentes de financiamiento que reciben los emprendimientos, denotando que más de la mitad $(51,3 \%)$ de los emprendimientos realizados por mujeres se sustentan en el apoyo bancario mediante créditos 0 préstamos. Además, el $63,5 \%$ de las participantes que han recibido un crédito financiero, lo han recibido del Banco Pichincha (figura 7). Por su parte, la gráfica 8 demuestra que las participantes consideran que el proceso para acceder a microfinanzas en las instituciones bancarias del país es favorable, puesto que el $27,5 \%$ y $44,7 \%$ señalan que el proceso para acceder a microcréditos es muy bueno y bueno, respectivamente. Según el GEM (2017) el apoyo financiero es un factor fundamental en el desarrollo y sostenibilidad de un emprendimiento, no obstante, en Ecuador este factor se encuentra 2,86 en una escala del 1 al 9; mientras que en países como Colombia y Perú se encuentra en 3,63 y 3,83, respectivamente. Se observa que Ecuador cuenta con un apoyo financiero por debajo del promedio para la región $(3,42)$, mientras que los demás países citados superan dicho promedio.

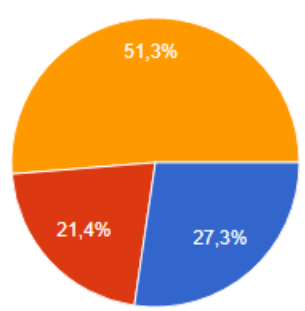

- Apoyo Familiar - Apoyo de mi Pareja Apoyo Bancario

Figura 6. Fuentes de financiamiento 


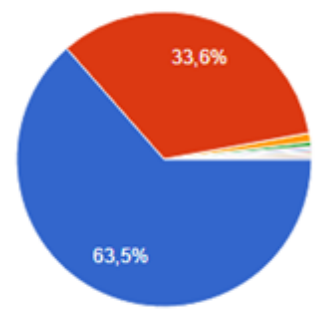

Figura 7. Acceso a microcréditos

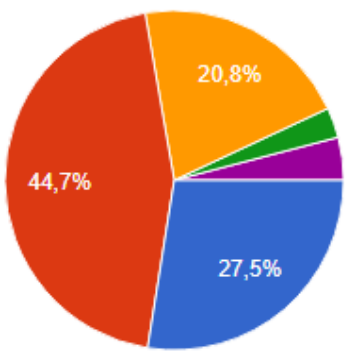

Figura 8. Proceso para otorgamiento de microfinanzas

\subsection{Emprendimiento y empoderamiento femenino}

La figura 9 muestra la percepción de las mujeres respecto a los factores que generan empoderamiento. Por tanto, las participantes señalan que el principal factor que provoca empoderamiento femenino es la independencia económica $(75,9 \%)$, seguido de la independencia familiar $(15,4 \%)$, y en menor magnitud el apoyo externo y las leyes 0 normativas gubernamentales. En este sentido, la figura 10 corrobora que el $88,7 \%$ de las mujeres considera que al independizarse de las fuentes económicas tradicionales alcanzan un estado de empoderamiento que les permite tomar decisiones y participar de forma activa y proactiva en su propio devenir. Cabe mencionar que según las cifras del Global Entrepreneurship Monitor, determinadas por Lasio et al., (2017), en Ecuador los emprendimientos entre hombres y mujeres no presentan diferencias significativas, puesto que la Tasa de Emprendimiento Temprano del 2017 para mujeres fue del $52 \%$, determinándose una relación del TEA femenino/masculino del 1,06, habiendo sido en el 2016: 0,9. Finalmente, en lo que respecta a la razón del emprendimiento, en los hombres predominan los emprendimientos por oportunidad y en las mujeres por necesidad.

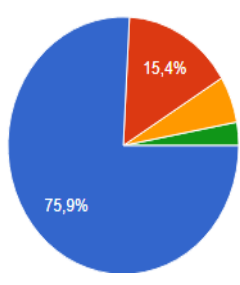

- Independencia económica

- Independencia fam

Apoyo externo

- Leyes o normativas gubernamentales

Figura 9. Factores que generan empoderamiento en la mujer 


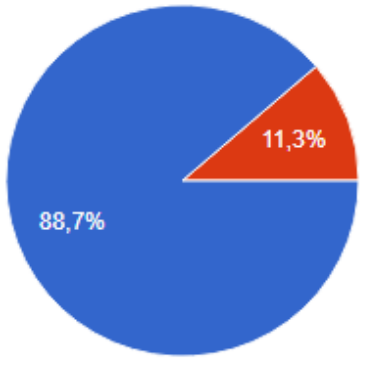

$\mathrm{S}$

No

Figura 10. Efectos del emprendimiento sobre la independencia económica de fuentes de ingresos tradicionales

Lo antes expuesto, demuestra que las mujeres consideran que la independencia económica les permite empoderarse y de este modo los emprendimientos tienen un rol relevante para mejorar las condiciones económicas de las mujeres, independientemente de su estatus económico. Kobeissi (2010) señala que las transformaciones que tienen lugar en el emprendimiento femenino permiten generar crecimiento económico y alivio de la pobreza, no obstante, ponen de relieve la necesidad de un análisis más global y diversificado de las actividades empresariales femeninas. Por otra parte, se menciona que las comparaciones internacionales de las prácticas empresariales femeninas siguen siendo limitadas lo que se incrementa en los países en vías de desarrollo. Este autor determinó que la educación femenina, el alcance de las actividades económicas femeninas, la proporción de ingresos femeninos, y la tasa de fertilidad son significativa en todas las estimaciones que evalúan el impacto de las actividades empresariales femeninas.

De forma similar, Haugh \& Talwar (2016) reportan que las normas sociales y culturales afectan en la participación de las mujeres en proyectos empresariales; en el norte de India se utilizaron datos de 49 miembros de una empresa social rural, en aras de evaluar la relación entre emprendimiento social, empoderamiento y cambio social. Los procesos empresariales innovadores que facilitaron la actividad económica de las mujeres y al mismo tiempo cumplieron con las normas sociales y culturales locales que limitan su agencia contribuyeron a cambiar el orden social en sí.

Se concluye que el emprendimiento social emancipatorio como proceso permite empoderar a las mujeres y paralelamente contribuye a cambiar el orden social en el que las mujeres se encuentran integradas.

Por otra parte, resulta importante contrastar las oportunidades y retos 
a los que se enfrentan los hombres y mujeres emprendedores. Kourilsky \& Walstad (1998) sugieren que la rápida expansión del reconocimiento de oportunidades creativas y el desarrollo de empresas comerciales demuestra que Estados Unidos está experimentando un cambio radical con respecto al espíritu empresarial. Aunque las tendencias y las proyecciones indican que las mujeres desempeñarán un papel cada vez más importante en el desarrollo empresarial de la economía, se sabe poco sobre lo que las jóvenes entienden o piensan sobre el espíritu empresarial. Impulsado por estas consideraciones subyacentes, este estudio investiga datos de encuestas de una muestra nacional de estudiantes de secundaria de ambos sexos sobre sus conocimientos y actitudes de emprendimiento, y si existen diferencias de género significativas en estas áreas. La Organización Gallup recolectó los datos del estudio de una muestra que incluyó aproximadamente 1.000 hombres y mujeres. Los resultados derivados del análisis de los datos sugieren que existen muchas similitudes entre mujeres y hombres con respecto a su conocimiento y opiniones sobre el espíritu empresarial; sin embargo, también exhiben diferencias de género significativas en varias áreas. Los resultados del estudio conducen a importantes implicaciones curriculares para la educación empresarial en las escuelas de la nación, especialmente en relación con las mujeres. Tanto hombres como mujeres exhiben un bajo nivel de conocimiento empresarial. Sin embargo, las mujeres son más conscientes de sus deficiencias en esta área de conocimiento que sus homólogos masculinos. Ambos sexos creen que la educación superior puede corregir el problema del conocimiento. Aunque están muy interesadas en iniciar un negocio, las mujeres aún son significativamente menos propensas que los hombres (62\% frente a $72 \%$ ) a querer iniciar un negocio propio. Tanto las mujeres como los hombres creen abrumadoramente en la importancia de retribuir a la comunidad, que va más allá de proporcionar empleos.

Además, en países como Indonesia el desarrollo de mujeres emprendedoras es una parte integral de las contribuciones económicas y el empoderamiento de las mujeres 
musulmanas. Por lo tanto, se realizó una investigación para determinar los desafíos a los que se enfrentan estas mujeres. Se concluyó que la calidad del emprendimiento empresarial y el éxito dependía en gran medida de las características personales de estas mujeres, en lugar de en cualquier sistema de educación o capacitación formal. Este estudio también descubrió que muchas mujeres mostraban estrategias de afrontamiento resilientes cuando trataban con fallas comerciales. Como consecuencia, pudieron prosperar a pesar de las restricciones sociales, culturales y políticas restrictivas.

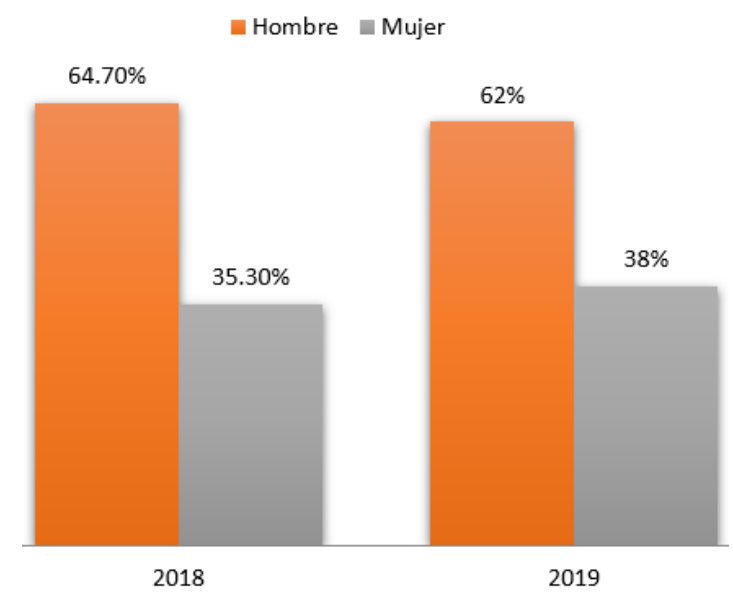

Figura 11. Microcréditos entregados por género

En el contexto local, en el año 2019, el Banco Guayaquil liquidó un total de 4.456 microcréditos en la provincia de Manabí, de los cuales el $62 \%$ se le otorgó a hombres y un
$38 \%$ a mujeres, si bien es cierto encontramos aun una brecha existente en la otorgación de microcréditos en relación al género, no obstante según los registros del Banco de Guayaquil, expuestos en la figura 11 se denota la disminución de esta brecha pasando de un $35,30 \%$ de microcréditos entregados a mujeres en el 2018 a un $38 \%$ en el año 2019 , los que genera una tasa de crecimiento anual del 2,7\%. Esto permite evidenciar que con el paso del tiempo, la mujer ha ido de a poco tomando un papel activo en el desarrollo productivo, esto se lo puede atribuir al sistema de microfinanzas empleado por las instituciones bancarias, que ha incidido en que la mujer pueda ser parte del sector financiero en el desarrollo económico familiar de los diferentes estratos poblacionales. De esta manera queda demostrado que los microcréditos no solo han ayudado a la mujer a empoderarse en el sector financiero, que años atrás eran acaparados casi en su totalidad por el género masculino, sino que también, ha pasado a formar parte del desarrollo productivo del país. En marzo del 2018 el volumen de crédito creció, estimando que el monto de crédito 
colocado en el segmento de Microcrédito por el Sistema Financiero llegaría a USD 4.645 millones; dentro de los cuales los Bancos Privados aportarían USD 1.461 millones, las COAC entregarían USD 2.290 millones, la Banca Pública aportaría USD 856 millones y las Mutualistas entregarían USD 37 millones. Según lo manifestado se determina una tasa de crecimiento promedio anual del sistema financiero de $28,75 \%$ en el volumen de crédito en monto, entre diciembre de 2002 y marzo de 2018 (Ocaña, 2018).

\section{Conclusiones}

Se destaca la importancia de las experiencias de mujeres emprendedoras en países en vías desarrollo y la necesidad imperiosa de integrar el desarrollo del emprendimiento femenino como un proceso que permita promover $y$ focalizar el empoderamiento de las mujeres. A pesar de las dificultades antes mencionadas, se demuestra que al independizarse de las fuentes económicas tradicionales (padres o parejas) las mujeres alcanzan un estado de empoderamiento que les permite tomar decisiones y participar de forma activa y proactiva en su propio devenir.

Por otra parte, se corrobora que las microfinanzas tienen una importancia relevante en el empoderamiento femenino, puesto que si bien han sido concebidas para generar desarrollo productivo; los grupos de mayor vulnerabilidad, en algunos casos, no podían acceder o en su defecto administrar asertivamente estas fuentes de financiamiento. Esto se debe a que las mujeres señalan, en su gran mayoría, que debido al emprendimiento que han liderado, se genera una satisfacción personal con una significancia altamente relacionada con el empoderamiento personal y colectivo.

La presente investigación demuestra como los microcréditos han contribuido al desarrollo productivo nacional, llegando a un monto de crédito estimado de USD 4.645 millones. Este sistema de créditos de microfinanzas ha logrado que la mujer tenga una participación activa en el sector económico, que a su vez se evidencia en los datos obtenidos del banco de Guayaquil a nivel de Manabí, que refleja una tasa de crecimiento anual de $2,7 \%$ en 
créditos otorgados a la mujer del año

2018 al 2019.

\section{Bibliografía}

Aguilar-Barojas, S. (2005). Fórmulas para el cálculo de la muestra en investigaciones de salud. Salud en Tabasco, 11(1-2), 333-338.

Banco Mundial. (2012). Guía para la regulación y la supervisión de las microfinanzas: Directrices de consenso. Washington, DC: Grupo Consultivo de Ayuda a los Pobres (CGAP).

Camino, R. C., \& Aguilar, A. E. (2017). Emprendimiento e innovación en Ecuador, análisis de ecosistemas empresariales para la consolidación de pequeñas y medianas empresas. INNOVA Research Journal, 2(9.1), 73-87. pp. 73-87. DOI: https://doi.org/10.33890/innov a.v2.n9.1.2017.504URL:

CEPAL. (2005). Un marco de análisis para el fomento de las políticas de desarrollo productivo con enfoque de género. Disponible en: https://repositorio.cepal.org/h andle/11362/5798

Colmenares, S. (2017). Análisis multivariable en geografía aplicado al estudio de la pobreza. Terra. Nueva Etapa, XXXIII (53), 41-73. [fecha de Consulta 24 de Junio de 2020]. ISSN: 1012-7089. Disponible

en: https://www.redalyc.org/articu lo.oa? id=721/72152384003

FAO. (2002). Servicios bancarios para los pobres: El microcrédito no basta, los sectores pobres necesitan otros servicios financieros [en línea]. "Cumbre Mundial sobre la Alimentación: cinco años después", Organización de las Naciones Unidas para la Agricultura y la Alimentación. Roma, 10 a 13 de agosto. Disponible en: http://www.fao.org/worldfoods ummit/spanish/newsroom/foc us/focus5.htm

García-Lobo, L. (2004). El desarrollo local como estrategia de desarrollo emergente en un mundo globalizado. Presente y Pasado. Revista de Historia, 9(17), 137-152.

GEM

(2015).

Global

Entrepreneurship Monitor. Ecuador -2015.

GEM.

(2017).

Global

Entrepreneurship Monitor. Ecuador 2016. ESPAE ESPOL.

Haugh, H. M., \& Talwar, A. (2016). Linking social entrepreneurship and social change: The mediating role of empowerment. Journal of Business Ethics, 133(4), 643658.

Iglesias, M. F. (2008). ¿Cómo potenciar el impacto social de las microfinanzas? El enfoque integral vs. el enfoque 
minimalista. El rol del Estado" en Renaud, Juliette y María F. Iglesias El impacto social de las microfinanzas. El caso de AVANZAR. Argentina: Centro de Estudios de la Estructura Económica-Universidad de Buenos Aires, pp. 24-72.

INEC. (2011). Encuesta de Estratificación del Nivel Socioeconómico NSE. Instituto Nacional de Estadísticas y Censos. Quito, Ecuador. Disponible en: https://www.ecuadorencifras. gob.ec//documentos/webinec/Estadisticas_Sociales/E ncuesta_Estratificacion_Nivel Socioeconomico/111220_N SE_Presentacion.pdf

INEC. (2018). Reporte de pobreza y desigualdad. Junio, 2018. Instituto Nacional de Estadísticas y Censos. Quito, Ecuador. Disponible en: https://www.ecuadorencifras. gob.ec/documentos/webinec/POBREZA/2018/Junio2018/Informe_pobreza_y_de sigualdad-junio_2018.pdf

INEC. (2019). Encuesta Nacional de Empleo, desempleo y subempleo (ENEMDU). Instituto Nacional de Estadísticas y Censos. Quito, Ecuador. Disponible en: https://www.ecuadorencifras. gob.ec/documentos/webinec/POBREZA/2019/Diciem bre2019/201912_PobrezayDesig ualdad.pdf
Kobeissi, N. (2010). Gender factors and female entrepreneurship: International evidence and policy implications. Journal of international entrepreneurship, 8(1), 1-35. https://doi.org/10.1007/s1084 3-010-0045-y

Kourilsky, M. L., \& Walstad, W. B. (1998). Entrepreneurship and female youth: Knowledge, attitudes, gender differences, and educational practices. Journal of Business venturing, 13(1), 77-88. https://doi.org/10.1016/S0883 -9026(97)00032-3

Lasio, V., Ordeñana, X., Caicedo, G., Samaniego, A., Izquierdo, E., Zambrano, J., Tenesaca, T. (2017).

Global Entrepreneurship Monitor. Ecuador 2017. Disponible en: http://espae.espol.edu.ec/wpcontent/uploads/documentos/ GemEcuador2017.pdf

Mballa, L. V. (2017). Desarrollo local y microfinanzas como estrategias de atención a las necesidades sociales: un acercamiento teórico conceptual. Revista mexicana de ciencias políticas $y$ sociales, 62(229), 101-127.

Ministerio de Economía y Finanzas de Perú. (2018). Desarrollo productivo empresarial. Disponible en: https://www.mef.gob.pe/es/no rmatividad-rp/en-relacion-ala-materiaremunerativa/desarrollo- 
productivo-

empresarial\#: :text=El\%20de

sarrollo\%20del\%20sector\%2

0empresarial,valor\%20agreg

ado\%20en\%20la\%20econom

$\%$ C3\%ADa content/uploads/sites/4/downl oads/2019/03/Revista-

Supervision-No.-3-Diciembre2018.pdf

Ocaña, E. (2018). Formalización de las microfinanzas y su impacto en el desarrollo del sistema financiero ecuatoriano. SUPER VISION, 8-12. Disponible en: http://estadisticas.superbanco s.gob.ec/portalestadistico/port alestudios/wp- 